\title{
Tensor-Based Match Pursuit Algorithm for MIMO Radar Imaging
}

\author{
Ping HUANG, Xin LI, Hui WANG \\ College of Automation, Harbin Engineering University, Nantong Street 145, Harbin, 150001, China \\ hppmonkeyking@163.com, Xin Li@hrbeu.edu.cn, Hui Wang@hrbeu.edu.cn \\ Submitted December 19, 2017 / Accepted April 21, 2018
}

\begin{abstract}
In MIMO radar, existing sparse imaging algorithms commonly vectorize the receiving data, which will destroy the multi-dimension structure of signal and cause the algorithm performance decline. In this paper, the sparsity characteristic and multi-dimension characteristic of signals are considered simultaneously and a new compressive sensing imaging algorithm named tensor-based match pursuit (TMP) is proposed. Firstly, MIMO radar tensor signal model is established to eliminate "dimension disaster". Then, exploiting tensor decomposition to process tensor data sets, tensor-based match pursuit is formulated for multi-dimension sparse signal recovery. Simulation results validates that the proposed method can accomplish high-resolution imaging correctly compared with conventional greedy sparse recovery algorithms. Additionally, under fewer snapshots condition, RMSE of proposed method is lower than other sparse recovery algorithms.
\end{abstract}

\section{Keywords}

MIMO radar, Compressive Sensing (CS), tensor decomposition, sparse imaging, greedy algorithm

\section{Introduction}

Mulitple-input multiple-output (MIMO) radar is an new emerging radar system that has been paid much attention recently. Compared with canonical phased-array radar, MIMO radar can transmit and receive independent waveforms via multiple antennas [1] and show advantage in resolution and better parameter identifiability [2].Recently, research on MIMO radar is mainly focused on waveform design, array configurations, imaging application, parameter estimation and otherwise [3]. In this paper, we focus on the colocated MIMO radar imaging issue.

For MIMO radar imaging issue, many data-independent approaches, such as delay-and-sum (DAS) (also called matched filtering) algorithm [4] have been proposed. But
DAS algorithms are constrained by low-resolution and high sidelobe level due to the imperfect orthogonality of the measure matrix. For the purpose of enhancing the imaging quality,compressed sensing technology is introduced. It can not only reduce the amount of observation data but also improve the resolution of imaging. The radar imaging technology using compressed sensing is called radar sparse imaging. In aerial radar imaging applications, targets such as aircraft and missiles can be characterized by a few scattering points for the imaging area, so sparse signal recovery technique can be used to provide more accurate target description. The undetermined system of equations is a problem to be solved by using optimization algorithms, and this problem commonly appears in sparse signal processing. The essence of sparse imaging is solving underdetermined equations through compressed sensing technology to focus pixel of imaging area [5]. Compared with DAS algorithms, sparse imaging algorithms can decrease data sampling rate and system complexity [6], [7]. Because of the spatial spectral domain filling, the imaging results can lead to higher sidelobe levels and lower resolution if the DAS algorithm is used directly. Furthermore, spare recovery algorithm with its inner super resolution has the potential to promote imaging performance. For instance, iterative adaptive algorithm (IAA) was proposed in [8] to provide more accurate sparse signal representation for MIMO radar sparse imaging. The IAA algorithm was proved to provide accurate estimation when the number of snapshots was low, but suffered from significantly computational burden compared to DAS algorithms. In [9], the radar sparse imaging is completed by the Bayesian learning frame method, which needs the prior knowledge of interesting area. In [10], a combination between compressed sensing technology and conventional DAS beamforming algorithm is presented, which can recovery the sparse signal well and has a high SINR lever, but the final imaging still subjected to low-resolution and high-sidelobers caused by delay-and-sum algorithms.

Moreover, the above sparse imaging algorithms commonly vectorize the received data, which will destroy the multi-dimension structure of signal and cause the algorithm performance decline, especially in lower snapshots and signal to ratio (SNR) condition. Tensor decomposition is a powerful 
tool for multidimensional signal processing. In [11], an angle estimation algorithm for MIMO radar in the presence of colored noise fields is presented, which make use of tensor decomposition to estimate DOA. In [12], method to recover low-rank tensors has been proposed to image human body. But in the above approaches, compressed sensing technology is not involved. As a consequence, computational burden is intolerable and the sparsity of objection is neglected. In [13], a hybrid matching pursuit method is proposed to enhancing the imaging quality in through-wall radar imaging (TWRI). But the imaging model is not suitable for MIMO radar imaging and fail to solve the "dimension disaster" problem.

In this paper, we combine tensor structure decomposition with sparse signal recovery technology for MIMO radar imaging and a new algorithm named tensor-based match pursuit (TMP) algorithm is proposed. MIMO radar tensor signal model is established to eliminate "dimension disaster" at first. Then, exploiting tensor decomposition to approximate tensor data sets. At last, aimed at the drawback of conventional greedy recovery colocated, proposed algorithm can reconstruct high-resolution sparse image correctly by combining the strength of OMP and SP algorithms.

Notation: We denote vectors and matrices by boldface lowercase and uppercase letters, respectively. $(\cdot)^{\mathrm{T}}$ denotes the transpose operation, $(\cdot)^{\mathrm{H}}$ denotes the conjugate transpose operation. $\operatorname{vec}(\cdot)$ denotes the vectorization operation, which means stacking the columns of a matrix on top of each other. Symbol $\otimes$ denotes kronecker product and symbol o denotes the outer product of vectors.

\section{MIMO Radar Tensor Signal Model}

Consider a colocated MIMO radar with $M$ closely spaced transmitting antennas and $N$ closely spaced receiving antennas. All the elements are omnidirectional. The positions of the $m$ th transmitter and the $n$th receiver are shown in a polar coordinate as $\left(R_{\mathrm{Tx}, m}, \varphi_{\mathrm{Tx}, m}\right)$ and $\left(R_{\mathrm{Rx}, m}, \varphi_{\mathrm{Rx}, m}\right)$, respectively. $\varphi_{\mathrm{Tx}, m}$ is the angle between transmitting antenna and $Y$ axis. Similarly, $\varphi_{\mathrm{Rx}, m}$ is the angle between receiving antenna and $Y$ axis. The geometry of MIMO radar imaging is shown in Fig.1. There are $K$ targets located at $\mathbf{r}_{k}=\left(x_{k}, y_{k}\right)$, and $\sigma\left(\mathbf{r}_{k}\right)$ denotes the scatter RCS coefficient. The range between $m$ th transmitter antenna and $k$ th scatter is denoted by $R_{\mathrm{Tx}, m}^{k}$ and the range between $n$th receiver antenna and $k$ th scatter is denoted by $R_{\mathrm{Rx}, m}^{k}$ similarly. The range between imaging area center and the antennas location baseline is denoted by $R_{0}$.

Assuming that orthogonality condition of transmitted signals holds here, the $m$ th transmitted signal is defined by

$$
S_{m}(t)=p_{m}(t) \exp \left(\mathrm{j} 2 \pi f_{\mathrm{c}} t\right)
$$

where $p_{m}(t)$ denotes the transmitted signal envelope, $f_{\mathrm{c}}$ denotes the carrier frequency.
The correlation function can by be written as

$$
R_{m m^{\prime}}(\tau)= \begin{cases}1, & m=m^{\prime} \& \tau=0 \\ 0, & \text { otherwise. }\end{cases}
$$

The echo corresponding to the $m$ th transmitter and the $n$th receiver is given as

$$
S_{n}(t)=\sum_{k=1}^{K} \sum_{m=1}^{M} \sigma\left(\mathbf{r}_{k}\right) S_{m}\left(t-\tau_{n, m}(k)\right)
$$

where $\tau_{n, m}(k)$ is the range delay from the $m$ th transmitter to the $k$ th scatter and then to the $n$th receiver. Under the far field assumption, we have $\left|\mathbf{r}_{k}\right| \ll R_{\mathrm{Tx}, m}$ and $\left|\mathbf{r}_{k}\right| \ll R_{\mathrm{Rx}, m}$, then the distance $R_{\mathrm{Tx}, m}^{k}$ from the $m$ th transmitter to the $k$ th scatter and the distance $R_{\mathrm{Rx}, n}^{k}$ from the $k$ th scatter to the $n$th receiver can be approximated by

$$
\begin{aligned}
& R_{\mathrm{Tx}, m}^{k} \approx R_{\mathrm{Tx}, m}+\mathbf{I}_{\mathrm{Tx}, m} \cdot \mathbf{r}_{k}, \\
& R_{\mathrm{Rx}, n}^{k} \approx R_{\mathrm{Rx}, n}+\mathbf{I}_{\mathrm{Rx}, n} \cdot \mathbf{r}_{k}
\end{aligned}
$$

where $\mathbf{I}_{\mathrm{Tx}, m}$ and $\mathbf{I}_{\mathrm{Rx}, n}$ denote the unit vector from the $m$ th transmitter to the $k$ th scatter and the unit vector from the $n$th receiver to the $k$ th scatter, respectively, and they can be written as

$$
\begin{aligned}
\mathbf{I}_{\mathrm{Tx}, m} & =\left(\sin \varphi_{\mathrm{Tx}, m}, \cos \varphi_{\mathrm{Tx}, m}\right), \\
\mathbf{I}_{\mathrm{Rx}, n} & =\left(\sin \varphi_{\mathrm{Rx}, n}, \cos \varphi_{\mathrm{Rx}, n}\right)
\end{aligned}
$$

then, we can get the approximation of $\tau_{n, m}(k)$

$$
\begin{aligned}
\tau_{n, m}(k) & =\frac{R_{\mathrm{Tx}, m}^{k}+R_{\mathrm{Rx}, n}^{k}}{\mathrm{c}} \\
& \approx \frac{R_{\mathrm{Tx}, m}+R_{\mathrm{Rx}, n}+\mathbf{I}_{\mathrm{Tx}, m} \mathbf{r}_{k}+\mathbf{I}_{\mathrm{Rx}, n} \mathbf{r}_{k}}{\mathrm{c}}
\end{aligned}
$$

where c denotes the electronmagnetic wave velocity, $R_{\mathrm{Tx}, m}$ and $R_{\mathrm{Rx}, n}$ are known fixed term.

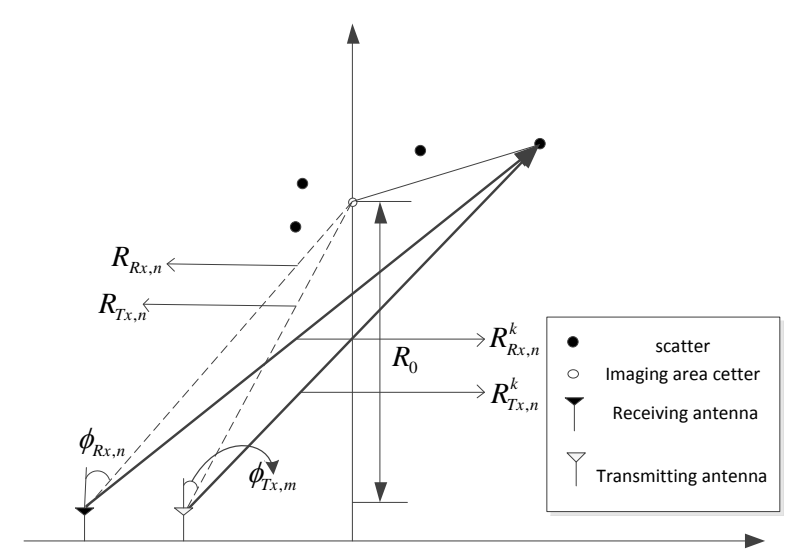

Fig. 1. Colocated MIMO radar imaging model. 
After matched filtering, the $(n, m)$ th channel signal is obtained, which can be written as

$$
\begin{aligned}
& S_{n, m}(t)=S_{n}(t) \otimes p_{m}^{*}(t) \\
&=\sum_{k=1}^{K} \sum_{m=1}^{M} \sigma\left(\mathbf{r}_{k}\right) p_{m}\left(t-\tau_{n, m}(k)\right) \\
& \exp \left(-\mathrm{j} 2 \pi f_{\mathrm{c}} \tau_{n, m}(k)\right) \otimes p_{m}^{*}(t) .
\end{aligned}
$$

Then, combining the route delay formula (6) and Fourier transform, the signal in frequency domain is given as

$$
z_{n . m}(f)=\sum_{k=1}^{K} \sigma\left(\mathbf{r}_{k}\right) \mathrm{e}^{-\mathrm{j} 2 \pi \frac{f_{\mathrm{c}}+f}{\mathrm{c}}\left(\mathbf{I}_{\mathrm{Tx}, m}+\mathbf{I}_{\mathrm{Rx}, n}\right) \mathbf{r}_{k}} .
$$

In (8), let

$$
\mathbf{K}_{n, m}(f)=-\frac{f_{\mathrm{c}}+f}{\mathrm{c}}\left(\mathbf{I}_{\mathrm{Tx}, m}+\mathbf{I}_{\mathrm{Rx}, n}\right)
$$

where $\mathbf{K}_{n, m}(f)=\left(K_{n, m}^{x}(f), K_{n, m}^{y}(f)\right)$ is the $(n, m)$ th channel wavenumber of MIMO radar. Then, the echo is given in wavenumber domain $\mathbf{K}_{n, m}(f)$ as

$$
z_{n . m}\left(\mathbf{K}_{n, m}(f)\right)=\sum_{k=1}^{K} \sigma\left(\mathbf{r}_{k}\right) \mathrm{e}^{\mathrm{j} 2 \pi \mathbf{K}_{n, m}(f) \mathbf{r}_{k}} .
$$

We rewrite (10) in discrete mode,

$$
\left\{\begin{array}{l}
z_{n . m}\left(\mathbf{K}_{n, m}\left(f_{i}\right)\right)=\sum_{k=1}^{V} \sigma\left(\mathbf{r}_{k}\right) \mathrm{e}^{\mathrm{j} 2 \pi \mathbf{K}_{n, m}\left(f_{i}\right) \mathbf{r}_{k}}, \\
f_{i}=\frac{B}{q} i, i=1, \cdots, q, \\
\mathbf{A}_{n, m}=\left[\begin{array}{lll}
\mathbf{a}_{n, m}(1) & \cdots & \mathbf{a}_{n, m}(K)
\end{array}\right] \\
\mathbf{a}_{n, m}(k)=\left[\begin{array}{lll}
\mathrm{e}^{\mathrm{j} 2 \pi \mathbf{K}_{n, m}\left(f_{1}\right) \mathbf{r}_{k}} & \cdots & \mathrm{e}^{\mathrm{j} 2 \pi \mathbf{K}_{n, m}\left(f_{q}\right) \mathbf{r}_{k}}
\end{array}\right]^{\mathrm{T}} \\
\sigma=\left[\begin{array}{lll}
\sigma\left(\mathbf{r}_{1}\right) & \cdots & \sigma\left(\mathbf{r}_{K}\right)
\end{array}\right]^{\mathrm{T}}
\end{array}\right.
$$

Formula (10) indicates that the target scatter coefficient and the echo in wavenumber domain of MIMO radar satisfy the relationship of Fourier transform. It's assumed that there are $Q$ samples in wavenumber domain in each channel. After matched filtering and Fourier transform, data of each sampling point is a matrix. Conventional matrix analysis algorithms vectorize every sampling data in (10) to compose a receiver data matrix, which ignore the multidimension structure of transmitting array, receiving array and sampling sequence. According to the definition of tensor, multiple samples of receiving data can be rewritten in tensor form. In another words, a tensor model $Z \in \mathbb{C}^{M \times N \times Q}$ can be builded using $Q$ samples. Based on the definition of tensor matrix-unfold, the three order tensor data $X$ can be written as

$$
[Z]_{(3)}^{\mathrm{T}}=A \sigma+N .
$$

Based on (12), the transpose of 3-mode product from MIMO radar tensor signal model $Z \in \mathbb{C}^{M \times N \times Q}$ equals the matrix form of receiver data. According the above analysis, MIMO radar tensor signal model consists of transmitting dimension, receiving dimension and sampling dimension, and tensor analysis algorithm can effectively exploit the multidimension characteristic information, which is ignored in conventional algorithm.

\section{MIMO Radar Imaging Technology Based on Multidimensional Com- pressed Sensing Algorithm}

On the basis of MIMO radar tensor signal model, we will first motivate the need of sparse tensor signal recovery in Sec. 3.1. The Tensor-based hybrid match pursuit algorithm will be introduced in Sec. 3.2.

\subsection{Introduction to Sparse Tensor Signal Re- covery}

In compressed sensing theory framework, the classical model is $\mathbf{z}=\boldsymbol{\Phi} \mathbf{y}$ with $\boldsymbol{\Phi} \in \mathbb{R}^{M \times I}$ and the equation is a underdetermined equation. If we want to use CS theory to process the tensor signal directly, the nature way is to vectorize the tensor signal and use typical greedy algorithms such as OMP and BP to recover the sparse vector and then reconstruct the original tensor signal. However, this strategy is quite unwise. The size of measurement matrix $\boldsymbol{\Phi}=\mathbf{B D} \in \mathbb{R}^{M \times I}$ will increase sharply as the tensor signal dimension increases. The computer burden increase as well.

To avoid the above shortcomings, tensor decomposition is introduced to approximate tensor signals. High-Order Singular Value Decomposition (HOSVD) [14] is a commonly tensor signal decomposition method. Specifically, it is defined by the following expression

$$
\underline{\mathbf{Y}}=\underline{\mathbf{X}} \times{ }_{1} \mathbf{D}_{1} \times{ }_{2} \mathbf{D}_{2} \cdots \times{ }_{N} \mathbf{D}_{N}
$$

where $\underline{\mathbf{X}} \in \mathbb{R}^{R_{1} \times R_{2} \times \cdots \times R_{n}}$ is core tensor and $\mathbf{D}_{n} \in \mathbb{R}^{I_{n} \times D_{n}}$ are unfolding matrices. The tensor decomposition schematic is shown in Fig.2.

In this paper, we consider HOSVD model and its application to solve compressed sensing problem, and exploit this method to fulfill MIMO radar sparse imaging.

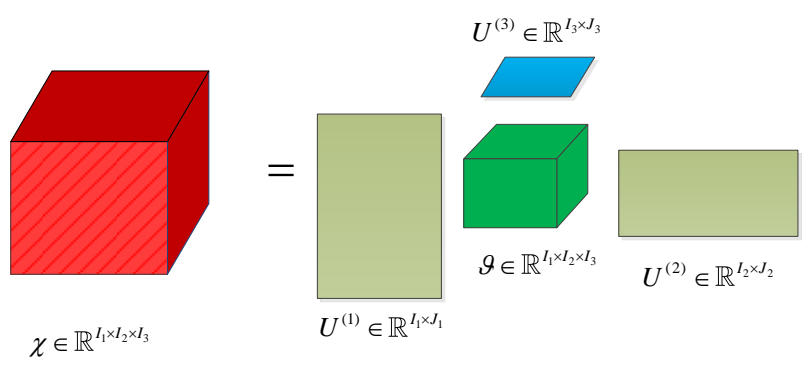

Fig. 2. Tensor decomposition schematic diagram. 


\subsection{Compressed Sensing Based on HOSVD De- composition}

As described in equation (13), the core MIMO radar tensor signal can be calculated by

$$
\underline{\mathbf{X}}=\underline{\mathbf{Y}} \times{ }_{1} \mathbf{D}_{1}^{\mathrm{T}} \times{ }_{2} \mathbf{D}_{2}^{\mathrm{T}} \times{ }_{3} \mathbf{D}_{3}^{\mathrm{T}} .
$$

Throughout linear measurements in each dimension, as follow

$$
=\begin{gathered}
\underline{\mathbf{Z}}=\underline{\mathbf{Y}} \times{ }_{1} \Phi_{1} \times{ }_{2} \Phi_{2} \times{ }_{3} \Phi_{3} \\
=\mathbf{X} \times{ }_{1} \Phi_{1} \mathbf{D}_{1} \times{ }_{2} \Phi_{2} \mathbf{D}_{2} \times{ }_{3} \Phi_{3} \mathbf{D}_{3}
\end{gathered}
$$

where $\boldsymbol{\Phi}_{i}(i=1,2,3)$ is the measurement matrix. Which can be also written in vectorized form as follow

$$
\mathbf{z}=\left(\mathbf{B}_{3} \otimes \mathbf{B}_{2} \otimes \mathbf{B}_{1}\right) \mathbf{x}, \quad \text { with }\|\mathbf{x}\|_{0} \leq \mathrm{K}
$$

where $\mathbf{z}=\operatorname{vec}(\underline{\mathbf{Z}})$ and $\mathbf{B}_{n}=\boldsymbol{\Phi}_{n} \mathbf{D}_{n}(n=1,2,3)$ and $K$ is the sparse degree. The tensor-based compressed sensing means to find the solution of a underdetermined equation with Kronecker structure.

\subsection{Tensor-Based Match Pursuit Algorithm}

The above analysis on tensor decomposition motivate us to propose the TMP algorithm. Through the TMP algorithm, the tensor decomposition is used to transform the receive signal into equation (17). In the proposed method, the standard OMP algorithm is used to solve the local sparse solutions, and the global estimation of the common support set is carried out by combination of all the local solution.

At the initialization stage, the sparse solution is calculated by using the Kron-OMP[16] algorithm, which can be expressed as

$$
\sigma_{\text {kron-OMP }}=\text { kron-OMP }\left(\underline{\mathbf{Z}}, \mathbf{B}_{1}, \mathbf{B}_{2}, \mathbf{B}_{3}, K\right)
$$

where $\sigma_{\text {kron-OMP }}$ is the solution of the Kron-OMP algorithm. Then the support set $\Lambda_{\text {old }}$ can be initialized as

$$
\Lambda_{\text {old }}=\text { max } \_ \text {ind }\left(\left|\sigma_{\text {kron-OMP }}\right|, K\right)
$$

where the function max_ind $(a, b)$ denotes $b$ indices corresonding to the largest magnitude entries in the vector $a$. The initial residual tensor is

$$
\underline{\mathbf{R}}=\underline{\mathbf{Z}}-\sum_{n=1}^{K} \sigma_{n} \mathbf{B}_{1}\left(\Lambda_{\text {old }}(n)\right) \circ \mathbf{B}_{2}\left(\Lambda_{\text {old }}(n)\right) \circ \mathbf{B}_{3}\left(\Lambda_{\text {old }}(n)\right)
$$

Then we still use Kron-OMP algorithm to process the residual

$$
\sigma_{\text {kron-OMP }}^{n}=\text { kron - OMP }\left(\underline{\mathbf{R}}, \mathbf{B}_{1}, \mathbf{B}_{2}, \mathbf{B}_{3}, K\right)
$$

where the function of kron-OMP denotes the output of KronOMP algorithm.
The support set is expanded to $2 \mathrm{k}$

$$
\Lambda_{\text {temp }}=\Lambda_{\text {old }} \cup \max \_ \text {ind }\left(\left|\sigma_{\text {kron-OMP }}^{n}\right|, K\right) .
$$

Then we can renew the support set

$$
\begin{aligned}
& \Lambda_{\text {new }}=\text { max_ind }\left(\underline{\mathbf{Z}} \times{ }_{3} \mathbf{B}_{1}\left(\Lambda_{\text {temp }}\right)^{\mathrm{T}}\right. \\
& \left.\times_{2} \mathbf{B}_{2}\left(\Lambda_{\text {temp }}\right)^{\mathrm{T}} \times{ }_{1} \mathbf{B}_{3}\left(\Lambda_{\text {temp }}\right)^{\mathrm{T}}, K\right) .
\end{aligned}
$$

The support set update is developed from SP algorithm. We use the equation (25) to renew the residual

$$
\underline{\mathbf{R}}=\underline{\mathbf{Z}}-\sum_{n=1}^{K} \sigma_{n} \mathbf{B}_{1}\left(\Lambda_{\text {new }}(n)\right) \circ \mathbf{B}_{2}\left(\Lambda_{\text {new }}(n)\right) \circ \mathbf{B}_{3}\left(\Lambda_{\text {new }}(n)\right) \text {. }
$$

Repeat renewing residual $\mathbf{R}$ and support set $\Lambda$, we can get the sparse solution.

\section{Simulation Result and Analysis}

In this section, simulations are performed to validate the proposed method. A colocated MIMO radar with $M=4$ transmit antennas and $N=4$ receive antennas is considered here. Both of them are arranged in half-wavelengthspaced uniform linear array, and the transmit and receive arrays are placed in $(0,4,8,12) \times \lambda / 2$ and $(0,1,2,3) \times \lambda / 2$ respectively. Transmitting orthogonal waveform is obtained by cycling algorithm-new algorithm (CAN) [15], in which the code number is 100 , carrier frequency is $10 \mathrm{GHz}$ and the bandwidth is $50 \mathrm{MHz}$. The corresponding code duration is $0.02 \mu \mathrm{s}$, the pulse repeat period is $6 \mu \mathrm{s}$, and the sample period equal the code time width. In order to compare the performance of the proposed algorithm, the results of Kron-OMP [16] and NBOMP [17] are also presented.

\subsection{Point Imaging Simulation}

Grid on the imaging zone. Considering that the observation area is divided in 50 range cells and the azimuth angel range from $-80^{\circ}$ to $80^{\circ}$. An gel cell is set $5^{\circ}$. Assuming that there are several point target in the imaging zone and the coordinations are $\left(40,0^{\circ}\right),\left(40,-20^{\circ}\right),\left(25,20^{\circ}\right),\left(5,-10^{\circ}\right)$, $\left(5,10^{\circ}\right),\left(10,10^{\circ}\right),\left(15,-10^{\circ}\right),\left(15,10^{\circ}\right)$ and $\left(45,10^{\circ}\right)$. We assume that all the radar cross section of the targets are 1 and the statistical characteristic of noise meet the additive gauss noise. The signal to noise ratio (SNR) is assumed $30 \mathrm{~dB}$. The imaging results of Kron-OMP algorithm, NBOMP algorithm and TMP algorithm are shown in Figs. 3-5. The fake shadows are marked with red circles on the Figs. 3-5.

From Figs. 3-5, we can conclude that all the three tensor-based algorithms can focus the correct imaging, but has the different performance. The imaging of KronOMP and NBOMP has fake shadow in incorrect place and the proposed algorithm can focus a radar imaging without fake shadow. 


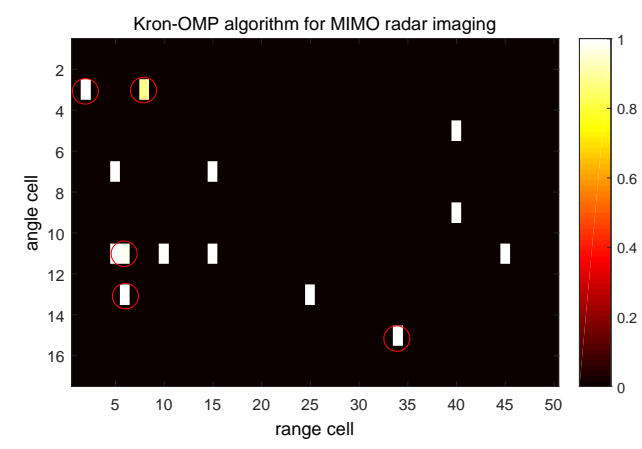

Fig. 3. Kron-OMP algorithm for MIMO radar imaging.

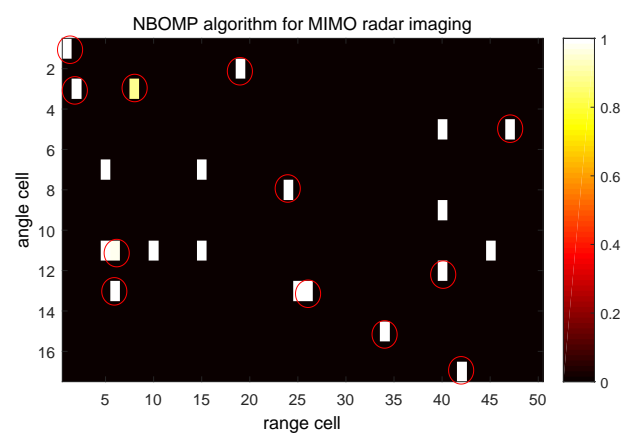

Fig. 4. NBOMP algorithm for MIMO radar imaging.

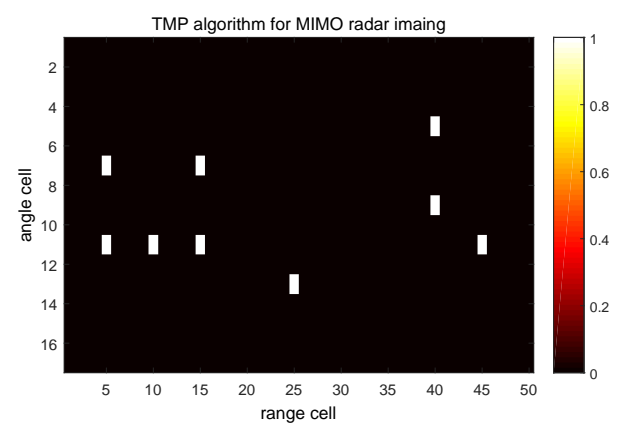

Fig. 5. TMP algorithm for MIMO radar imaging.

\subsection{TMP Algorithm Performance Analysis}

In this example, we will demonstrate the performance of the introduced algorithms described in Sec. 3 by using the parameter of root mean square error (RMSE). Results are shown in Fig. 6 and Fig. 7, in which percentage of correctly recovered tensor versus SNR is presented. In order to effectively explain the proposed algorithm superiority, standard OMP and SP are presented at the same time. Gradient algorithm for ISAR proposed in [18] and a new 2D signals recovering algorithm proposed in [19] are presented at the same time to compare with the TMP algorithm. From Fig. 6 and Fig. 7, we can conclude that all the seven algorithms' performance become better with SNR increasing. The performance of three algorithm based on tensor structure are better than the vectorization-based algorithm. The reason is that operation of vectorizing the received data destroy the multidimension structure of signal and cause the multidimension information loss. Meanwhile, The performance of proposed algorithm is better than the others, because the proposed method combines the index selection strategy of OMP and the index reevaluation strategy of SP based on tensor signal model. TMP can reconstruct high-resolution radar image with no artifacts compared with the other two tensor-based algorithms.

We compared the operation time and reconstruction accuracy of the above seven algorithms. The simulations are performed in MATLAB R2015a environment using an Inter ${ }^{\circledR}$ Core $^{\mathrm{TM}} \mathrm{i} 7-6700 \mathrm{HQ}, 2.6 \mathrm{GHz}$ processor with $8 \mathrm{~GB}$ of memory, and under Microsoft Windows 10 operating system. The SNR is set $15 \mathrm{~dB}$. The results are shown in Tab. 1 .

From Tab. 1, we can conclude that compared with other conventional greedy algorithms, the proposed TMP method can get better sparse recovery accuracy at the cost of increasing computering burden. The reason is that the tensor structure of the received data is applied in the proposed TMP algorithm, and tensor decomposition operation can suppress the additive noise. As a consequence, the RMSE can decrease while the CPU time increase due to tensor decomposition.

Then, the relationship between snapshots and RMSE is demonstrated by mathematical simulations. The result is shown in Fig. 8. The answer reveals that the error will decrease along with snapshots increase, and the performance of proposed algorithm is better than the others.

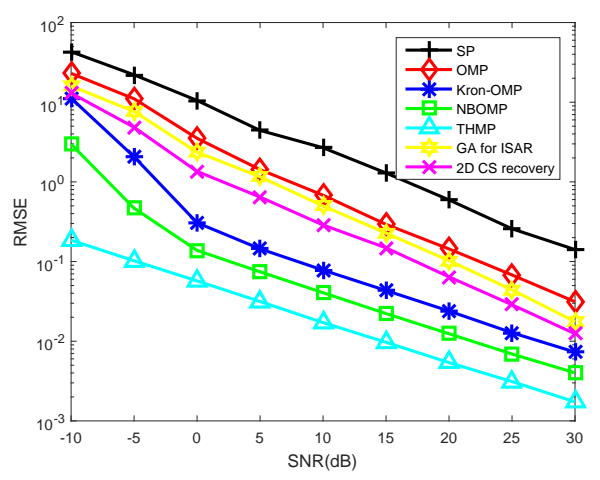

Fig. 6. RMSE vesus SNR of five algorithm.

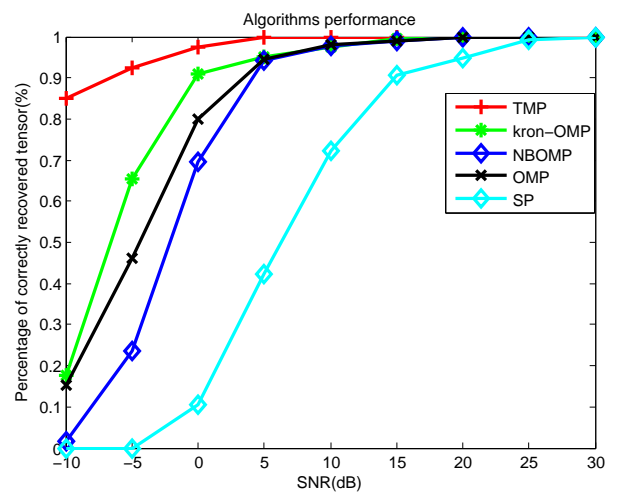

Fig. 7. Accurate recovery probability versus SNR. 


\begin{tabular}{|c|c|c|c|c|c|c|c|}
\hline Recovery algorithm & OMP & SP & Kron-OMP & NBOMP & TMP & GA for ISAR & 2D CS Recovery \\
\hline Running time $[\mathrm{s}]$ & 2.148 & 3.654 & 11.245 & 10.476 & 10.833 & 3.465 & 6.735 \\
\hline RMSE & 0.2927 & 1.2364 & 0.0433 & 0.0222 & 0.0097 & 0.2276 & 0.1485 \\
\hline
\end{tabular}

Tab. 1. Performance comparison of different methods.

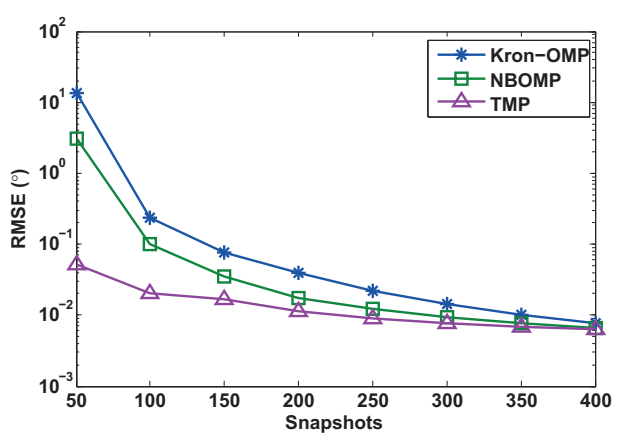

Fig. 8. The relationship between snapshots and RMSE.

\section{Conclusion}

In this paper, a new Tensor-based Match Pursuit algorithm (TMP) for MIMO radar imaging is proposed. We extend the tensor decomposition technology to MIMO radar sparse imaging. MIMO radar signal is firstly formulated as a tensor-based sparse model. Then, the aim of MIMO radar sparse imaging is recoverying the sparse signal in tensor formulor. By combining the advantage of OMP and the advantage of SP in back tracking strategy, the proposed TMP algorithm can reconstructs high-precision radar images without distortion. In colored noise field, high order cross correlation matrix tensor decomposition algorithm is proposed in this paper. This approach can be used to mitigate the performance loss of TMP algorithm caused by colored noise. Simulation results validate the performance of the proposed method.

\section{Acknowledgments}

This research was supported by the National Natural Science Foundation $(61571148,61403091)$, China postdoctoral special funding (2015T80328), China Postdoctoral Science Foundation Grant (2014M550182), Heilongjiang Natural Science Foundation (QC2015049), Heilongjiang Postdoctoral Special Fund (LBH-TZ0410, LBH-Q16065) and Innovation of Science and Technology Talents in Harbin (2013RFXXJ016), Research and development project of application technology in Harbin (2017RAQXJ095).

\section{References}

[1] FISHLER, E., HAIMOVICH, A., et al. MIMO radar: an idea whose time has come. In Proceedings of the 2004 IEEE Radar Conference. Philadelphia (USA), 2004, p. 71-78. DOI: $10.1109 /$ NRC.2004.1316398
[2] FRANKIE, K. W., SO, H. C., et al. Parameter estimation and identifiability in bistatic multiple-input multiple-output radar. IEEE Transactions on Aerospace and Electronic Systems, 2015, vol. 51, no. 3, p. 2047-2056. DOI: 10.1109/TAES.2015.130502

[3] DENG, H., GENG, Z., et al. MIMO radar waveform design for transmit beamforming and orthogonality. IEEE Transactions on Aerospace and Electronic Systems, 2016, vol. 52, no. 3, p. 1421-1433. DOI: 10.1109/TAES.2016.140023

[4] ZHUGE, X. D., YAROVOY, A. G., et al. Modified Kirchhoff migration for UWB MIMO array-based radar imaging. IEEE Transactions on Geoscience and Remote Sensing, 2010, vol. 48, no. 6, p. 26922703. DOI: 10.1109/TGRS.2010.2040747

[5] YOON, Y. S., AMIN, M. G. Compressed sensing technique for highresolution radar imaging. In Proceedings of the SPIE Defense and Security Symposium (DSS'08), 2008. DOI: 10.1117/12.777175

[6] DING, L., CHEN, W. D. MIMO radar sparse iamging with phase mismatch. IEEE Geoscience and Remote Sensing Letters, 2015, vol. 12, no. 4, p. 816-820. DOI: 10.1109/LGRS.2014.2363110

[7] SADEQUE, A., ALI, T., et al. Waveform transmission scheme for MIMO radar imaging based on space-time block codes. IEEE Transactions on Aerospace and Electronic Systems, 2014, vol. 50, no. 1, p. 777-785. DOI: 10.1109/TAES.2013.120226

[8] ROBERTS, W., STOCIA, P., et al. Iterative adaptive approaches to MIMO radar imaging. IEEE Journal of Selected Topics in Singal Processing,IEEE Journal of Selected Topics in Signal Processing, 2010, vol. 4, no. 1, p. 5-20. DOI: 10.1109/JSTSP.2009.2038964

[9] WU, Q., ZHANG, Y. D., et al. Multi-static passive SAR imaging based on Bayesian compressive sensing. In Proceedings of the SPIE - The International Society for Optical Engineering, 2014. DOI: $10.1117 / 12.2050524$

[10] TANG, V. H., BOUZERDOUM, A., et al. Two-stage through-the-wall radar image formulation using compressive sensing. Journal of Electronic Imaging, 2013, vol. 22, no. 2. DOI: 10.1117/1.JEI.22.2.021006

[11] WANG, X. P., WANG, W., et al. Sparsity-aware DOA estimation scheme for noncircular source in MIMO radar. Sensors, 2016, vol. 16, no. 4, p. 113. DOI: $10.3390 /$ S16040539

[12] CAIAFA, C. F., CICHOCKI, A. Computing sparse representations of multidimensional signals using kronecker bases. Neural computation, 2013, vol. 25, no. 1, p. 186-220. DOI: 10.1162/NECO_a_00385

[13] LI, G., BURKHOLDER, R. J. Hybrid matching pursuit for distributed through-wall radar imaging. IEEE Transactions on Antennas and Propagation, 2015, vol. 63, no. 4, p. 1701-1711. DOI: 10.1109/TAP.2015.2398115

[14] CAIAFA, C. F., CICHOCKI, A. Multidimensional compressed sensing and their applications. Wiley Interdisciplinary Reviews: Data Mining and Knowledge Discovery, 2013, vol. 3, no. 6, p. 355-380. DOI: 10.1002/WIDM.1108

[15] HE, H., STOICA, P., LI, J. Designing unimodular sequence sets with good correlations-including an application to MIMO radar. IEEE Transactions on Signal Processing, 2009, vol. 57, no. 11, p. 4391-4405. DOI: 10.1109/TSP.2009.2025108

[16] CAIAFA, C. F., CICHOCKI, A. Block sparse representations of tensors using Kronecker bases. In Proceedings of the IEEE International Conference on Acoustics, Speech and Signal Processing (ICASSP). Kyoto (Japan), 2012, p. 2709-2712. DOI: 10.1109/ICASSP.2012.6288476 
[17] ELDAR, Y., KUPPINGER, P., BOLCSKEI, H. Block-sparse signals: uncertainty relations and efficient recovery. IEEE Transactions on Signal Processing, 2010, vol. 58, no. 6, p. 3042-3054. DOI: 10.1109/TSP.2010.2044837

[18] DAKOVIC, M., STANKOVIC, L., et al. Gradient algorithm based ISAR image reconstruction from the incomplete dataset. In Proceedings of the 3rd International Workshop on Compressed Sensing Theory and its Applications to Radar, Sonar and Remote Sensing (CoSeRa 2015). Pisa (Italy), 2015, p. 6-10. DOI: 10.1109/CoSeRa.2010.2044837

[19] STANKOVIC, S., OROVIC, I. An approach to 2D signals recovering in compressive sensing context. Circuits, Systems, and Signal Processing, 2017 , vol. 36, no. 4, p. 1700-1713. DOI :10.1007/s00034016-0366-8
About the Authors ...

Ping HUANG was born in 1980. He received his $\mathrm{Ph} . \mathrm{D}$. degree from Harbin Engineering University, Harbin, China in 2011. His research interests include radar signal processing and integrated navigation system.

Xin LI was born in 1981. He received his Ph.D. degree from Harbin Engineering University, Harbin, China in 2012. His research interests include radar signal processing.

Hui WANG was born in 1963. He received his B.S. degree from Harbin Engineering University, Harbin, China in 2006. His research interests include radar signal processing. 